Tropical Journal of Pharmaceutical Research June 2019; 18 (6): 1305-1313

ISSN: $1596-5996$ (print); 1596-9827 (electronic)

(c) Pharmacotherapy Group, Faculty of Pharmacy, University of Benin, Benin City, 300001 Nigeria.

\title{
Gas chromatography-mass spectrometry analysis of principal lipid-soluble components of Pinellia ternate fermented with Bacillus subtilis, Aspergillus niger and Meyerozyma guillermondii
}

\author{
Lin Wang ${ }^{1}$, Jiabin Sun ${ }^{1}$, Meiying Yue ${ }^{2}$, Jin Wang ${ }^{1 *}$, Qinwan Huang ${ }^{1}$, Zelun Lan ${ }^{3}$ \\ ${ }^{1}$ College of Pharmacy, ${ }^{2}$ College of Basic Medicine, Chengdu University of Traditional Chinese Medicine, No. 1166, Liutai Road, \\ Wenjiang District, Chengdu 611137, ${ }^{3}$ Sichuan Neautus Traditional Chinese Medicine Co. Ltd, No. 8 South Herui Road, High- \\ Tech Zone, Chengdu 611731, China
}

*For correspondence: Email: wangjin0816@126.com

Sent for review: 19 December 2018

Revised accepted: 12 May 2019

\begin{abstract}
Purpose: To study the differences in lipid-soluble compounds from naturally-fermented Rhizoma Pinelliae fermentata (BXQ) samples, and fermentation products of $B X Q$ using pure cultures of Bacillus subtilis, Aspergillus niger, and Meyerozyma guillermondii.

Methods: First, unfermented BXQ (CTFJ-Q), traditional, naturally-fermented BXQ (CTFJ-H), and fermentation products of BXQ using pure cultures of Bacillus subtilis (XJFJ), Aspergillus niger (MJFJ), and Meyerozyma guillermondii (JMJFJ) were obtained. Their lipid-soluble components were then analyzed using gas chromatography-mass spectrometry (GC-MS) technology and principal component analysis (PCA).

Results: GC-MS results showed that there were 26, 24, 27, 31 and 32 types of chemical components in CTFJ-Q, CTFJ-H, XJFJ, MJFJ and JMJFJ, respectively. Furthermore, PCA revealed that samples obtained using fermentation with pure cultures of the three microorganisms had unique chemical components.

Conclusion: These results suggest that the microorganisms used for fermentation greatly influence the lipid-soluble components of BXQ. This finding is considered beneficial for the optimization of $B X Q$ fermentation process.
\end{abstract}

Keywords: BanXia Qu, Pure culture fermentation, Pinellia ternate, Principal component analysis, Lipidsoluble components

This is an Open Access article that uses a fund-ing model which does not charge readers or their institutions for access and distributed under the terms of the Creative Commons Attribution License (http://creativecommons.org/licenses/by/4.0) and the Budapest Open Access Initiative (http://www.budapestopenaccessinitiative.org/read), which permit unrestricted use, distribution, and reproduction in any medium, provided the original work is properly credited.

Tropical Journal of Pharmaceutical Research is indexed by Science Citation Index (SciSearch), Scopus, International Pharmaceutical Abstract, Chemical Abstracts, Embase, Index Copernicus, EBSCO, African Index Medicus, JournalSeek, Journal Citation Reports/Science Edition, Directory of Open Access Journals (DOAJ), African Journal Online, Bioline International, Open-J-Gate and Pharmacy Abstracts

\section{INTRODUCTION}

Banxia Qu (BXQ), also called Rhizoma Pinelliae fermentata, a fermented product of Pinellia ternate Thunb. Breit with ginger juice and flour, is a popular traditional herbal medicine used for resolving phlegm, relieving cough and enhancing digestion in Traditional Chinese Medicinal theory $[1,2]$. Medicinal fermentation refers to a metabolic process that produces chemical 
changes in medical material through the action of enzymes, thereby enhancing existing therapeutic effects, or generating new curative effects $[3,4]$.

Since natural fermentation process is not regulated, too many microorganisms are involved in the process, leading to instability in quality control $[5,6]$. Therefore, it is necessary to conduct research on fermentation of $B X Q$ using pure cultures to replace the traditional natural fermentation process, so as to generate $B X Q$ samples with stable qualities for clinical use.

Based on 16S r DNA and 26S r DNA sequence analyses, previous investigations have revealed that the dominant bacteria identified during the traditional and natural fermentation for $B X Q$ were Streptomyces sp., Bacillus pumilus, B. subtilis, $B$. aryabhattai and Bacillus sp. Furthermore, the dominant yeast was Meyerozyma guilliermondii, while the dominant molds were Paecilomyces variotii, Byssochlamys spectabilis and Aspergillus niger [7]. Studies have also found that the main active chemical components were digestive enzymes, volatile oils, glycyrrhizic acid and glycyrrhetinic acid [1]. In addition, a previous study found that after the fermentation of $P$. ternata, a clear aroma was produced, which may be related to changes in lipid-soluble substances in the fermentation process.

In the present study, GC-MS technology combined with PCA was used to determine differences in lipid-soluble components between $B X Q$ derived from traditional natural fermentation, components derived from fermentation using pure $B$. subtilis, $A$. niger, and M. guillermondii.

\section{EXPERIMENTAL}

\section{Preparation of fermented material}

Pinelliae Rhizoma Praeparatum Cum Alumine and alum were purchased from Sichuan Neautus Traditional Chinese Medicine Co. (Chengdu, China). Liushenqu was provided by Sichuan Fuzheng Pharmaceutical Co. (Jianyang, China). As shown in Figure 1, it comprised $160 \mathrm{~g}$ of alum of processed pinellia, $10 \mathrm{~g}$ of Angelica alum, $5 \mathrm{~g}$ of Liushenqu, $20 \mathrm{~g}$ of ginger juice, and $32 \mathrm{~g}$ of flour. The fine powder was stirred well with ginger juice and made into granules [2]. Then, a pre-fermentation sample was taken and labeled as CTFJ-Q. Then, the prepared materials were incubated in a constant temperature and humidity chamber for 3 days at $37^{\circ} \mathrm{C}$ and relative humidity of $75 \%$, to produce "yellow clothing", which was then freeze-dried. The natural fermented sample of $B X Q$ was obtained and marked as CTFJ-H. The prepared material was sterilized at $121^{\circ} \mathrm{C}$ for $20 \mathrm{~min}$. After cooling, it was used for pure-breed fermentation.

\section{Preparation of spore suspension}

Bacillus subtilis CICC 10066, Aspergillus niger CICC 2039, Meyerozyma guillermondii CICC 32608 which were purchased from China Center of Industrial Culture Collection, were inoculated and cultured on beef extract peptone medium, potato culture medium, and wort agar medium, respectively.

Single colonies were taken up and inoculated on their appropriate medium plates, then sub-

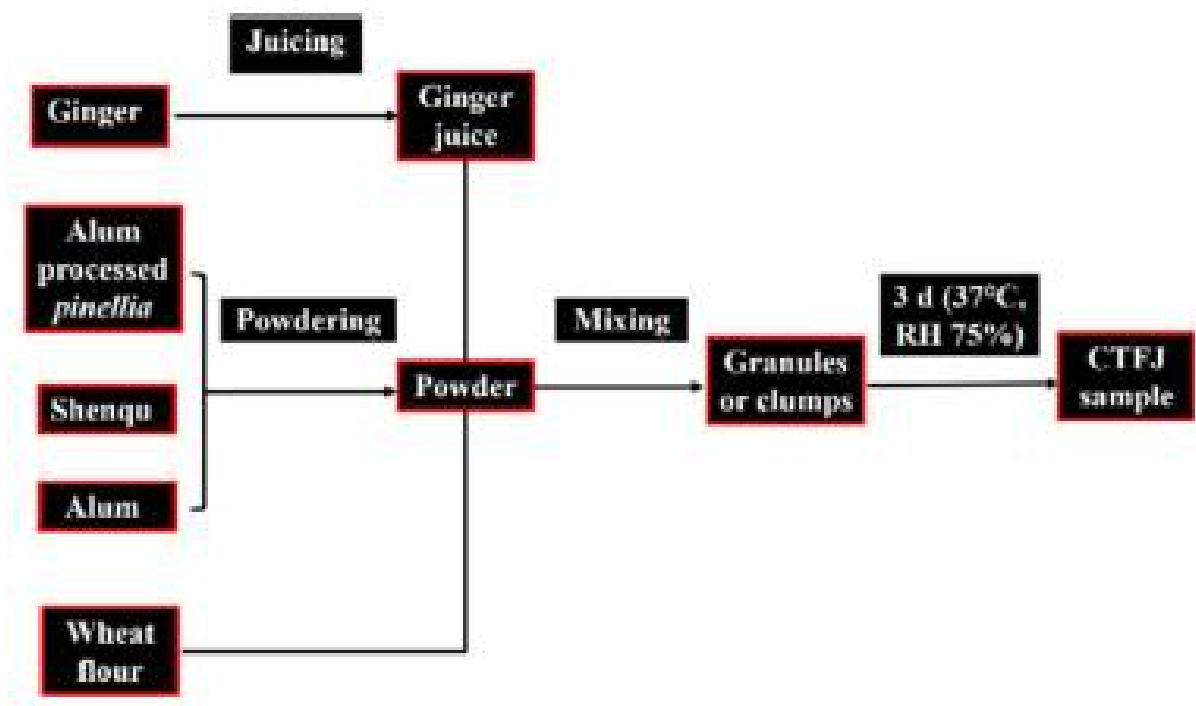

Figure 1: $B X Q$ traditional fermentation process 
cultured in either 28 or $37^{\circ} \mathrm{C}$ incubator until pure cultures were obtained. The pure cultures were washed with sterile water and the spores on the slants were washed and diluted to a spore suspension of $2 \times 10^{5}$ spores $/ \mathrm{mL}$.

\section{Pure fermentation}

The spore suspension was separately inoculated into sterilized material at a volume of $6 \mathrm{~mL}$ per bottle under aseptic conditions, and incubated in a constant temperature and humidity chamber for 3 days at $37{ }^{\circ} \mathrm{C}$ and relative humidity of $75 \%$. After the fermentation, the koji was taken out and freeze-dried. Then, samples were obtained and designated as follows: $B$. subtilis sample (XJFJ), A. niger sample (MJFJ), and $M$. guillermondii sample (JMJFJ). The pure fermentation process is shown in Figure 2.

\section{GC-MS analysis}

\section{GC-MS analysis conditions}

The GC/MS analysis was performed on an Agilent 7890A gas chromatograph (Agilent Technologies, Santa Clara, CA, USA) connected to an Agilent 5975C mass spectrometer (Agilent Technologies, Santa Clara, CA, USA). The chromatographic column was a DB-5MS column (30 $\mathrm{m} \times 0.25 \mathrm{~mm} \times 0.25 \mu \mathrm{m}$ film thickness).

The operation was performed under the following conditions: He carrier gas at a flow rate of 1.0 $\mathrm{mL} / \mathrm{min}$; injection temperature was $280^{\circ} \mathrm{C}$; initial column temperature of was $60^{\circ} \mathrm{C}$, and increased to $200{ }^{\circ} \mathrm{C}$ at a ramp rate of $20{ }^{\circ} \mathrm{C} / \mathrm{min}$, and thereafter ramped to $280{ }^{\circ} \mathrm{C}$ at the rate of 10 ${ }^{\circ} \mathrm{C} / \mathrm{min}$. Split injection was applied with volume of $1.0 \mu \mathrm{L}$ and split ratio of 50:1. Solvent was delayed by $2.0 \mathrm{~min}$. The scanning range was from 35 to 550 amu, and the mass spectrometer was operated in electron impact (El) mode with the electron energy set at $70 \mathrm{eV}$. Each compound was identified using the National Institute of Standards and Technology (NIST) library (14.L).

\section{Preparation of sample solution}

Each sample (20 g material) was crushed into fine powder which could go through 80 mesh sieve, and then refluxed with $200 \mathrm{~mL}$ of petroleum ether (boiling range $=60-90^{\circ} \mathrm{C}$ ) for 3 h. The solution was evaporated to dryness under reduced pressure at $70{ }^{\circ} \mathrm{C}$, and the residue was dissolved in chloroform to yield a concentration of $4 \mathrm{mg} / \mathrm{mL}$.

\section{Data processing}

Chromatographic peaks were recognized and identified using the NIST14.L MS data library. The relative content (RC) was measured with single constituent area (SA) and total area (TA) was calculated as shown in Eq (1).

$R C=(S A / T A) 100$

PCA was carried out using SIMCA-P software (version 11.0, Umetrics, Umea, Sweden).

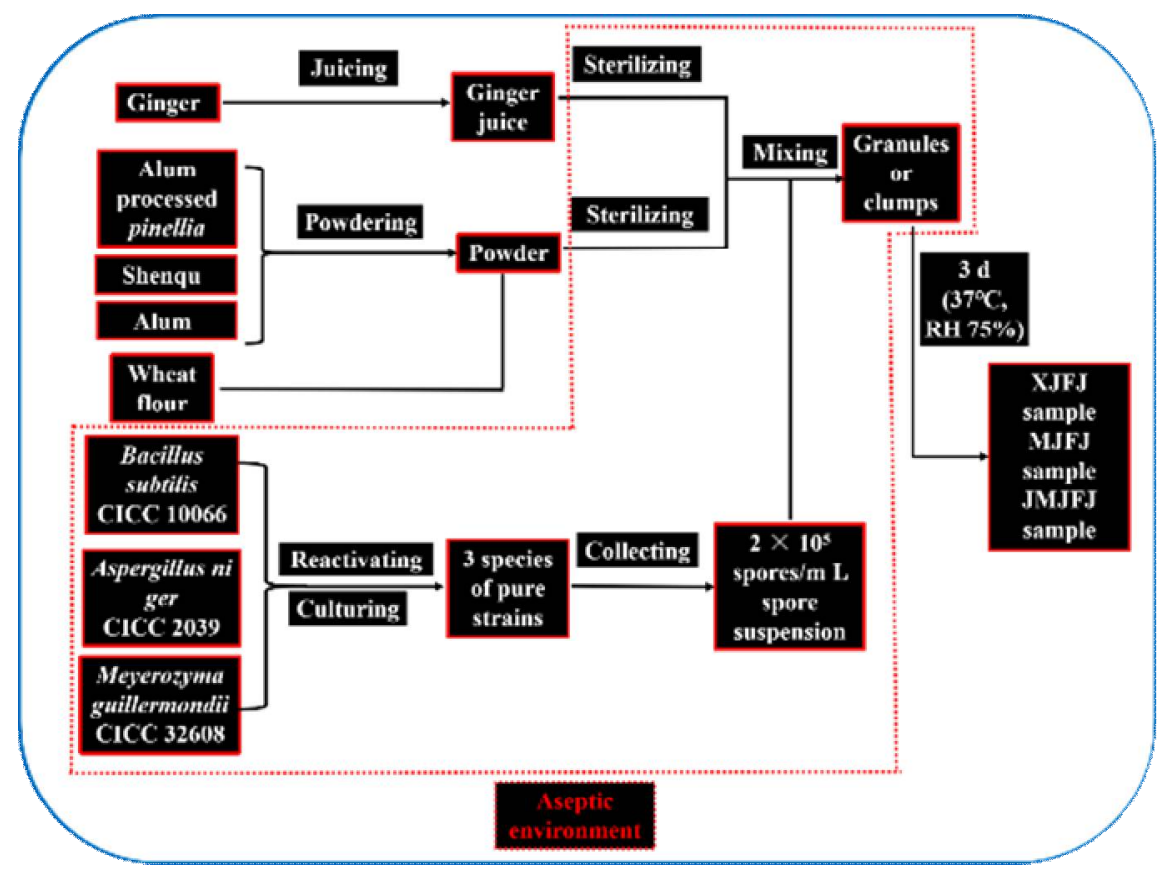

Figure 2: $B X Q$ pure fermentation process 


\section{RESULTS}

\section{Chromatographic results}

The relative content of each component as determined using GC-MS was calculated by area normalization method and referred to related literature research results (Table 1$)$. The results showed that a total 46 components were detected in lipid-soluble components of the five $\mathrm{BXQ}$ samples, and 26, 24, 27, 31, 32 chemical constituents were identified from the CTFJ-Q, CTFJ-H, XJFJ, MJFJ and JMJFJ samples, respectively. The typical GC-MS total ion chromatogram (TIC) of fat-soluble components in the five BXQ samples are shown in Figure 3. The lipid-soluble components of $\mathrm{BXQ}$ were mainly alkenes, sterols, organic acids, esters, aldehydes, phenols, and ketones. The most abundant components were acids and sterols.

Alkenes: A total of 9 alkanes were detected from the five $B X Q$ samples in the present study. Cycloeicosane and eicosane were unique components of the CTFJ-Q samples. Hexadecane and heptadecane were unique components of the MJFJ samples. Octadecane was detected in XJFJ and MJFJ samples; henecicosane was detectable only in MJFJ and JMJFJ samples, while cyclododecane, cycloeicosane and nonacosane were present in all $B X Q$ samples.

Olefins: Trans- $\alpha$-bergamotene was detectable in the XJFJ and MJFJ samples but not in the other three samples. The other samples contained curcumene but it was not in the XJFJ and MJFJ samples. This change might be the effect of different microbial fermentations on the composition of $B X Q$.

Sterols: A total of 7 sterol compounds were identified in the five $B X Q$ samples, representing $11.50-18.70 \%$ of the total content of the lipidsoluble components. Docosanol was detected only in the MJFJ sample; paradol was detectable only in the CTFJ-H samples, while isofucosterol was detected only in the XJFJ samples. Stigmasterol, $\beta$-Sitosterol, fucosterol and cycloartenol were present in all 5 samples. $\beta$ Sitosterol was highest in relative content. The relative content of $\beta$-sitosterol in XJFJ sample was $13.12 \%$, followed by stigmasterol with relative content of $2.85 \%$ in $\mathrm{CTFJ}-\mathrm{H}$; then cycloartenol, with relative content of $2.56 \%$ in CTFJ-Q. The least was fucosterol, with relative content of $2.21 \%$ in CTFJ-H.

Organic acids: There were obvious differences in the total acids of the five samples. The highest was the MJFJ samples, with relative content of $21.24 \%$, followed by the JMJFJ samples, with relative content of $16.28 \%$. The contents of acids in CTFJ-Q and XJFJ samples were relatively close, i.e. 12.54 and $13.37 \%$, respectively, and the least was CTFJ-H with a relative content of $5.52 \%$.

Esters: Fermentation by different microorganisms only affected the relative content of dibutyl phthalate and ethyl linoleate. Methyl palmitate, ethyl linoleate, and (5-methylheptyl) 3-(4methoxyphenyl)-2-propenoate were produced after Pinellia pure fermentation.

Aldehydes: Increases in aldehydes after fermentation resulted in clear aroma. The E-15heptadecenal was detected in all samples except CTFJ-Q. 2, 4-Decadienal and (E, E)- 2,4decadienal were also detected in JMJFJ samples.

Phenols: 2, 4-di-tert-butylphenol was detected in all five samples, indicating that the samples before and after fermentation were not degraded. Due to the influence of fermentation process, gingerols disappeared in traditional fermentation and pure-culture fermentations.

Ketones: 4-campestene-3-one was a unique component of the XJFJ samples; zingerone was unique to the CTFJ-Q samples, while 4-(1, 5dimethyl-4-hexenyl)-2-cyclohexen-1-one was unique to JMJFJ.

Other components: The relative content of erucylamide in the CTFJ-Q and CTFJ-H samples was higher than that in the other three groups, while 2, 6-di-tert-butyl-p-benzoquinone was present only after fermentation.

\section{PCA results}

The PCA was conducted, taking the relative content of the 46 components as the dependent variables, and 5 groups of samples as variables. The score and loading plots of triplicate results are shown in Figure 4 and Figure 5, respectively. All samples were successfully divided into five groups in the score plot, with no overlap. As can be seen from Figure 5, numbers 9 (zingiberene), 14 (zingiberone), 18 (1-octadecene), 25 (cycloeicosane), 35 (eicosane), 37 (10-shogaol), 38 (erucylamide) and 46 (handianol) were the major components of CTFJ-Q; number 30 (paradol) contributed more to CTFJ-H; while numbers 13 (hexadecane), 15 (heptadecane) and 34 (1-eicosene) were the main components of MJFJ. 
Table 1: Lipid-soluble compositions of Pinellia ternate (Thunb.) Breit fermented with different fermentation processes

\begin{tabular}{|c|c|c|c|c|c|c|c|c|}
\hline \multirow[t]{2}{*}{ No. } & \multirow[t]{2}{*}{ RT } & \multirow[t]{2}{*}{ Compound } & \multirow[t]{2}{*}{ MS match $/ \%$} & \multicolumn{5}{|c|}{ Relative percentage content (\%) } \\
\hline & & & & CTFJ-Q & CTFJ-H & XJFJ & MJFJ & JMJFJ \\
\hline 1 & 4.093 & Behenic alcohol & 91 & $0.00 \pm 0.00$ & $0.00 \pm 0.00$ & $0.00 \pm 0.00$ & $0.00 \pm 0.00$ & $0.28 \pm 0.02$ \\
\hline 2 & 4.575 & Cyclododecane & 96 & $0.44 \pm 0.02$ & $0.27 \pm 0.05$ & $0.25 \pm 0.03$ & $0.43 \pm 0.12$ & $0.45 \pm 0.02$ \\
\hline 3 & 5.351 & 2,4-Decadienal & 91 & $0.00 \pm 0.00$ & $0.00 \pm 0.00$ & $0.00 \pm 0.00$ & $0.00 \pm 0.00$ & $0.24 \pm 0.01$ \\
\hline 4 & 5.516 & $(\mathrm{E}, \mathrm{E})-2,4-$ Decadienal & 94 & $0.00 \pm 0.00$ & $0.00 \pm 0.00$ & $0.00 \pm 0.00$ & $0.00 \pm 0.00$ & $0.40 \pm 0.01$ \\
\hline 5 & 5.981 & 1-Tetradecene & 98 & $1.17 \pm 0.02$ & $0.94 \pm 0.02$ & $1.02 \pm 0.10$ & $0.96 \pm 0.06$ & $1.55 \pm 0.02$ \\
\hline 6 & 6.587 & 2,6-Di-tert-butyl-p-benzoquinone & 98 & $0.00 \pm 0.00$ & $0.18 \pm 0.03$ & $0.06 \pm 0.02$ & $0.16 \pm 0.01$ & $0.28 \pm 0.01$ \\
\hline 7 & 6.663 & Curcumene & 96 & $0.26 \pm 0.01$ & $0.28 \pm 0.03$ & $0.00 \pm 0.00$ & $0.00 \pm 0.00$ & $0.22 \pm 0.01$ \\
\hline 8 & 6.734 & trans-.a.-Bergamotene & 97 & $0.00 \pm 0.00$ & $0.00 \pm 0.00$ & $0.15 \pm 0.02$ & $0.06 \pm 0.01$ & $0.00 \pm 0.00$ \\
\hline 9 & 6.746 & Zingiberene & 90 & $0.25 \pm 0.01$ & $0.00 \pm 0.00$ & $0.00 \pm 0.00$ & $0.00 \pm 0.00$ & $0.00 \pm 0.00$ \\
\hline 10 & 6.816 & 2,4-Di-tert-butylphenol & 91 & $2.25 \pm 0.01$ & $1.69 \pm 0.05$ & $1.86 \pm 0.03$ & $1.66 \pm 0.08$ & $2.98 \pm 0.04$ \\
\hline 11 & 6.940 & $\beta$-sesquiphellandrene & 96 & $0.32 \pm 0.00$ & $0.12 \pm 0.02$ & $0.13 \pm 0.03$ & $0.13 \pm 0.00$ & $0.13 \pm 0.02$ \\
\hline 12 & 7.263 & Cetene & 98 & $1.40 \pm 0.02$ & $1.12 \pm 0.07$ & $1.35 \pm 0.04$ & $0.98 \pm 0.06$ & $1.95 \pm 0.05$ \\
\hline 13 & 7.304 & Hexadecane & 93 & $0.00 \pm 0.00$ & $0.00 \pm 0.00$ & $0.00 \pm 0.00$ & $0.18 \pm 0.01$ & $0.00 \pm 0.00$ \\
\hline 14 & 7.751 & Zingiberone & 93 & $0.19 \pm 0.01$ & $0.00 \pm 0.00$ & $0.00 \pm 0.0$ & $0.00 \pm 0.00$ & $0.00 \pm 0.00$ \\
\hline 15 & 7.945 & Heptadecane & 95 & $0.00 \pm 0.00$ & $0.00 \pm 0.00$ & $0.00 \pm 0.00$ & $0.13 \pm 0.01$ & $0.00 \pm 0.00$ \\
\hline 16 & 8.028 & 4-(1,5-dimethyl-4-hexenyl)-2-Cyclohexen-1- & 94 & $0.00 \pm 0.00$ & $0.00 \pm 0.00$ & $0.00 \pm 0.00$ & $0.00 \pm 0.00$ & $0.11 \pm 0.01$ \\
\hline 17 & 8.357 & Tetradecanoic acid & 93 & $0.00 \pm 0.00$ & $0.00 \pm 0.00$ & $0.08 \pm 0.01$ & $0.07 \pm 0.01$ & $0.10 \pm 0.01$ \\
\hline 18 & 8.587 & 1-Octadecene & 99 & $1.06 \pm 0.01$ & $0.00 \pm 0.00$ & $0.00 \pm 0.00$ & $0.00 \pm 0.00$ & $0.00 \pm 0.00$ \\
\hline 19 & 8.575 & E-15-Heptadecenal & 99 & $0.00 \pm 0.00$ & $0.83 \pm 0.02$ & $1.07 \pm 0.10$ & $0.75 \pm 0.02$ & $1.54 \pm 0.05$ \\
\hline 20 & 9.051 & Pentadecanoic acid & 99 & $0.00 \pm 0.00$ & $0.00 \pm 0.00$ & $0.14 \pm 0.02$ & $0.17 \pm 0.01$ & $1.54 \pm 0.05$ \\
\hline 21 & 9.522 & Methyl hexadecanoate & 99 & $0.00 \pm 0.00$ & $0.00 \pm 0.00$ & $0.19 \pm 0.01$ & $0.07 \pm 0.02$ & $0.08 \pm 0.01$ \\
\hline 22 & 9.828 & n-Hexadecanoic acid & 99 & $3.88 \pm 0.02$ & $2.03 \pm 0.03$ & $4.54 \pm 0.16$ & $7.67 \pm 0.10$ & $10.02 \pm 0.05$ \\
\hline 23 & 9.880 & Dibutyl phthalate & 90 & $1.39 \pm 0.02$ & $0.70 \pm 0.04$ & $0.98 \pm 0.05$ & $1.66 \pm 0.04$ & $1.56 \pm 0.02$ \\
\hline 24 & 10.022 & 1-Nonadecene & 99 & $1.41 \pm 0.01$ & $1.43 \pm 0.01$ & $0.97 \pm 0.01$ & $1.33 \pm 0.05$ & $1.54 \pm 0.03$ \\
\hline 25 & 10.034 & Cycloeicosane & 99 & $0.87 \pm 0.01$ & $0.00 \pm 0.00$ & $0.00 \pm 0.00$ & $0.00 \pm 0.00$ & $0.00 \pm 0.00$ \\
\hline 26 & 10.822 & 9,12-Octadecadienoic acid (Z,Z)-, methyl & 99 & $0.00 \pm 0.00$ & $0.00 \pm 0.00$ & $0.36 \pm 0.03$ & $0.36 \pm 0.05$ & $0.17 \pm 0.02$ \\
\hline 27 & 11.198 & $(Z, Z)-9,12-O c t a d e c a d i e n o i c ~ a c i d ~$ & 99 & $8.66 \pm 0.02$ & $3.49 \pm 0.38$ & $8.61 \pm 0.18$ & $13.33 \pm 6.77$ & $4.61 \pm 0.24$ \\
\hline
\end{tabular}

RT, Retention time 
Table 1: Lipid-soluble compositions of Pinellia ternate (Thunb.) Breit fermented with different fermentation processes (continued)

\begin{tabular}{|c|c|c|c|c|c|c|c|c|}
\hline \multirow[t]{2}{*}{ No. } & \multirow[t]{2}{*}{ RT } & \multirow[t]{2}{*}{ Compound } & \multirow[t]{2}{*}{ MS match $/ \%$} & \multicolumn{5}{|c|}{ Relative percentage content (\%) } \\
\hline & & & & CTFJ-Q & CTFJ-H & XJFJ & MJFJ & JMJFJ \\
\hline 29 & 11.433 & (5-Methylheptyl)3-(4-methoxyphenyl)-2-propenoate & 91 & $0.00 \pm 0.00$ & $0.00 \pm 0.00$ & $0.00 \pm 0.00$ & $1.24 \pm 0.09$ & $1.27 \pm 0.04$ \\
\hline 30 & 11.998 & Paradol & 90 & $0.00 \pm 0.00$ & $0.47 \pm 0.03$ & $0.00 \pm 0.00$ & $0.00 \pm 0.00$ & $0.00 \pm 0.00$ \\
\hline 31 & 13.098 & Cycloeicosane & 97 & $0.96 \pm 0.02$ & $0.66 \pm 0.04$ & $0.33 \pm 0.03$ & $1.08 \pm 0.11$ & $0.78 \pm 0.02$ \\
\hline 32 & 13.886 & Octadecane & 95 & $0.00 \pm 0.17$ & $0.00 \pm 0.06$ & $0.31 \pm 0.10$ & $1.40 \pm 0.09$ & $0.00 \pm 0.00$ \\
\hline 33 & 14.180 & Diacetoxy-6-gingerdiol & 91 & $0.31 \pm 0.02$ & $0.44 \pm 0.04$ & $0.00 \pm 0.03$ & $0.00 \pm 0.11$ & $0.00 \pm 0.02$ \\
\hline 34 & 14.604 & 1-Eicosene & 94 & $0.00 \pm 0.00$ & $0.00 \pm 0.00$ & $0.34 \pm 0.02$ & $1.53 \pm 0.11$ & $0.41 \pm 0.03$ \\
\hline 35 & 14.645 & Eicosane & 93 & $1.54 \pm 0.04$ & $0.00 \pm 0.00$ & $0.00 \pm 0.00$ & $0.00 \pm 0.00$ & $0.00 \pm 0.00$ \\
\hline 36 & 15.386 & Heneicosane & 93 & $0.00 \pm 0.00$ & $0.00 \pm 0.00$ & $0.00 \pm 0.00$ & $1.12 \pm 0.05$ & $1.15 \pm 0.02$ \\
\hline 37 & 15.639 & 10-Shogaol & 94 & $1.03 \pm 0.07$ & $0.00 \pm 0.00$ & $0.00 \pm 0.00$ & $0.00 \pm 0.00$ & $0.00 \pm 0.00$ \\
\hline 38 & 16.121 & Erucylamide & 94 & $1.59 \pm 0.02$ & $1.26 \pm 0.24$ & $0.47 \pm 0.06$ & $0.95 \pm 0.03$ & $0.86 \pm 0.01$ \\
\hline 39 & 16.661 & Squalene & 96 & $1.42 \pm 0.04$ & $1.60 \pm 0.06$ & $1.41 \pm 0.10$ & $1.69 \pm 0.01$ & $1.24 \pm 0.04$ \\
\hline 40 & 17.180 & Nonacosane & 94 & $0.56 \pm 0.09$ & $1.15 \pm 0.02$ & $0.49 \pm 0.03$ & $0.59 \pm 0.07$ & $0.75 \pm 0.04$ \\
\hline 41 & 23.138 & Stigmasterol & 94 & $2.31 \pm 0.04$ & $2.85 \pm 0.06$ & $1.87 \pm 0.02$ & $1.46 \pm 0.06$ & $1.54 \pm 0.12$ \\
\hline 42 & 24.427 & $\mathrm{y}$-Sitosterol & 99 & $10.47 \pm 0.05$ & $11.55 \pm 0.23$ & $13.12 \pm 0.13$ & $7.85 \pm 0.15$ & $9.99 \pm 0.06$ \\
\hline 43 & 24.856 & Fucosterol & 96 & $1.72 \pm 0.03$ & $2.21 \pm 0.07$ & $0.00 \pm 0.00$ & $0.76 \pm 0.04$ & $2.11 \pm 0.05$ \\
\hline 44 & 24.885 & Isofucosterol & 91 & $0.00 \pm 0.00$ & $0.00 \pm 0.00$ & $2.18 \pm 0.01$ & $0.00 \pm 0.00$ & $0.00 \pm 0.00$ \\
\hline 45 & 25.362 & 4-Campestene-3-one & 92 & $0.00 \pm 0.00$ & $0.00 \pm 0.00$ & $1.10 \pm 0.03$ & $0.00 \pm 0.00$ & $0.00 \pm 0.00$ \\
\hline 46 & 26.303 & Handianol & 99 & $2.56 \pm 0.02$ & $1.58 \pm 0.07$ & $0.73 \pm 0.08$ & $1.44 \pm 0.12$ & $1.29 \pm 0.09$ \\
\hline
\end{tabular}

RT, Retention time 


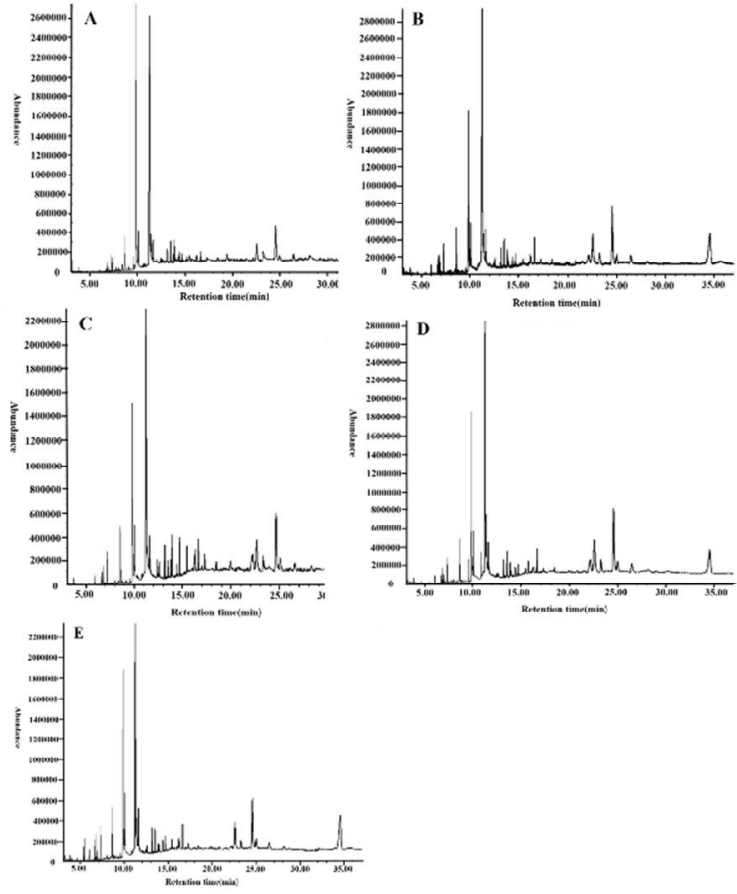

Figure 3: TICs of 5 representative samples via (GCMSA-CTFJ-Q, B-CTFJ-H, C-XJFJ, D-MJFJ, E-JMJFJ)

For XJFJ, the principal components were numbers 8 (trans- $\alpha$-bergamotene), 44 (isofucosterol) and 45 (4-campestene-3-one), while numbers 1 (behenic alcohol), 3 (2,4decadienal), 4 (E,E-2,4-decadienal), 16 (4-(1,5dimethyl-4-hexenyl)-2-cyclohexen-1-one), 20 (pentadecanoic acid) and 22 (n-hexadecanoic acid) were most prominent in JMJFJ. Nevertheless, the complete separation of all BXQ samples indicated that each class had its own unique lipid-soluble profile.

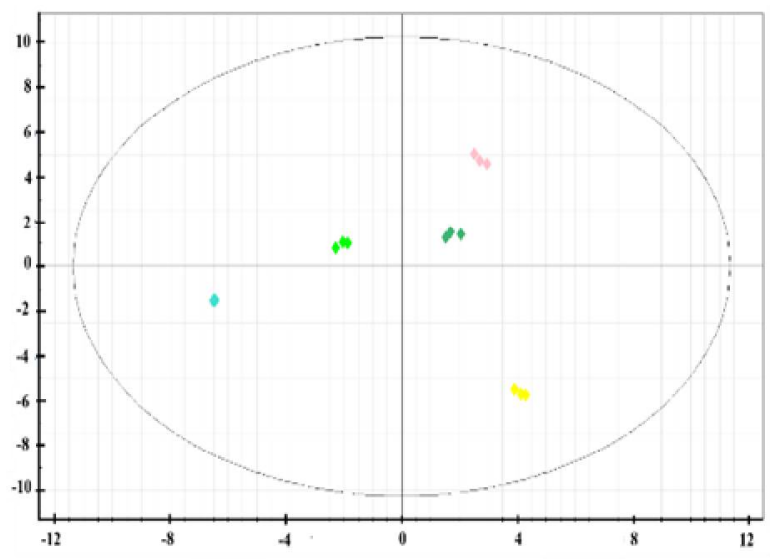

Figure 4: PCA principal component score plot

\section{DISCUSSION}

Previous studies showed that Rhizoma Pinelliae fermentata exhibited antitussive, expectorant, antiemetic, antitumor, anti-bacterial, anti- inflammatory, antioxidant, and sedative properties [8-10]. However, not much is known about the microorganisms in Rhizoma Pinelliae fermentata and the chemical compositions of volatile oils resulting from its fermentation with pure cultures of $B$. subtilis, $A$. niger, and $M$. guillermondii. Therefore, in this study, GC-MS and PCA were used to determine the differences in lipid-soluble compounds derived from traditional natural fermentation and the pureculture fermentation of $\mathrm{BXQ}$ with $B$. subtilis, $A$. niger, and $M$. guillermondii.

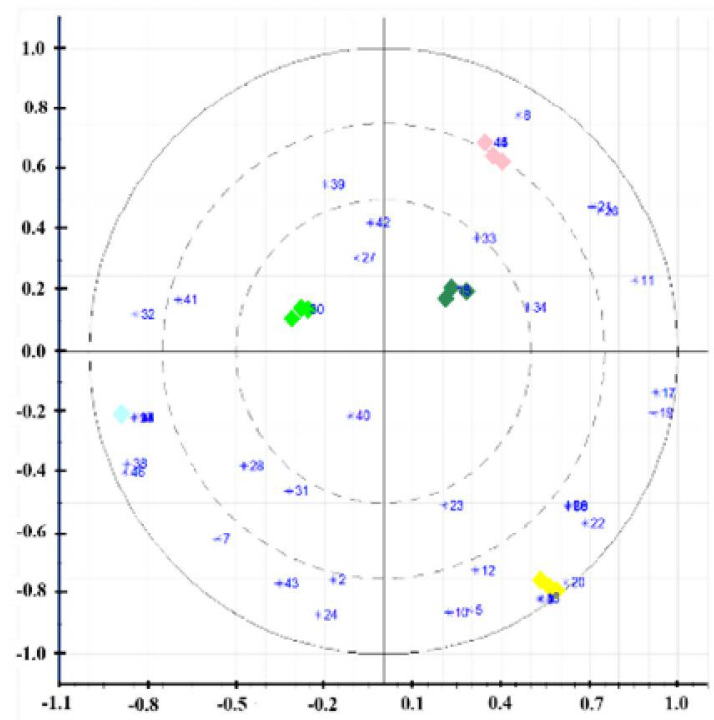

Figure 5: Score and loading plots of triplicate results

The results from GC-MS revealed that the chemical composition changed significantly after fermentation. The types and relative contents of sterols varied greatly among the five samples. $\beta$ Sitosterol and isofucosterol exert cholesterollowering effects. Isofucosterol was detected only in the XJFJ sample which contained the highest amount of $\beta$-sitosterol, suggesting that $B$. subtilis-fermented product may exert anti-obesity effects. The fermentation product of $M$. guillermondii had the highest amount of conjugated linoleic acids. These compounds improve menopausal symptoms, prevent obesity and increase beneficial bacteria in the intestine [11-13].

Methyl palmitate, ethyl linoleate, and 5methylheptyl, 3-(4-methoxyphenyl)-2-propenoate were produced after pure culture fermentation. Studies have shown that the lipid-soluble components of phytolacca such as dibutyl phthalate, methyl palmitate, methyl linoleate and linoleic acid have positive effects on the large intestine [14]. Thus, the results of pure culture fermentation were better than those of the CTFJ$\mathrm{H}$ samples in terms of the appearance of methyl palmitate and ethyl linoleate. Aldehydes have 
delicate fragrance. Increases in the concentration of aldehydes after fermentation resulted in clear aroma. It has been reported that 2, 4-di-tertbutylphenol reduced the spoilage of cereals through its anti-fungal and anti-oxidant properties [15]. It can also be used for treating group $A$ Streptococcus (GAS) infection through inhibition of the growth of biofilms [16].

All 5 samples yielded 2, 4-di-tert-butylphenol, indicating that the samples before and after fermentation were not easily degraded. However, 2, 6-di-tert-butyl-p-benzoquinone was detected only after fermentation, indicating that the fermentation process resulted in the conversion of phenolic compounds into terpenoids.

According to principal component analysis, the closer the variables to the corresponding samples, the greater the probability of contribution of these samples. All tested samples were successfully divided into five individual groups. The fermented samples were found to possess unique characteristic aroma, when compared with non-fermented samples, indicating that fermentation was an indispensable and active process. Different strains of the fermented samples and the traditionally fermented samples also had significantly different compositions, indicating that Bacillus subtilis, Aspergillus niger and Meyerozyma guillermondii play important roles in the fermentation process.

\section{CONCLUSION}

The results show that microorganisms have strong influences on the lipid-soluble components of BXQ samples. The present study is the first to make a comparison between the products of traditional, natural fermentation of $B X Q$, and products obtained using pure culture fermentation, and the results provide a theoretical basis for the process of preparation of Rhizoma Pinelliae fermentata. Moreover, the results indicate that the quality of Rhizoma Pinelliae fermentata can be determined by measuring the contents of these four dominant bacteria. However, it is not certain how the microorganisms influence the production of the chemical compounds, or whether pure microbial cultures can replace the traditional natural fermentation of $B X Q$. Thus, there is a need for further studies on processing technology, to investigate the relationship between microorganisms and Rhizoma Pinelliae fermentata in vivo and in vitro.

\section{DECLARATIONS}

\section{Conflict of interest}

No conflict of interest is associated with this work.

\section{Contribution of authors}

We declare that this work was done by the author(s) named in this article and all liabilities pertaining to claims relating to the content of this article will be borne by the authors. L. W., J. S., Q. H., J. W. conceived and designed the study, M. Y. and Z. L. collected and analysed the data, L. W., J. S., Q. H., J. W. wrote the manuscript. All authors read and approved submission of the manuscript for publication.

\section{Open Access}

This is an Open Access article that uses a funding model which does not charge readers or their institutions for access and distributed under the terms of the Creative Commons Attribution License (http://creativecommons.org/licenses/by/ 4.0) and the Budapest Open Access Initiative (http://www.budapestopenaccessinitiative.org/rea d), which permit unrestricted use, distribution, and reproduction in any medium, provided the original work is properly credited.

\section{REFERENCES}

1. Zhang $C$, Zhao $C, X u M, L U Y$, Huang $Q$. Reviews on Historical Evolution and Modern Research of Rhizoma Pinelliae Fermentata. World Sci Tech/Moder Trad Chin Med Mater Med 2015; 17: 1893-1898.

2. Chinese Pharmacopoeia Commission. Traditional Chinese Medicine Prescription Preparation. Beijng: People's medical Publishing House 1995: 49.

3. Zhu H, Xie W, Long K. Study on dynamic changing regularity of microflora in fermentation process of Sojae Semen Praeparatum by PCR-DGGE. ChinTradit Herbal Drugs 2017; 48: 1757-1765.

4. Zhang $L X$, Gao WY, Wang $H Y$. Review of traditional Chinese medicine processed by fermentation. China $J$ Chin Mater Med 2012; 37: 3695-3700.

5. Wang LY, Cheong KL, Wu DT, Meng LZ, Zhao J, Li SP. Fermentation optimization for the production of bioactive polysaccharides from Cordyceps sinensis fungus UM01. Int J Biol Macromol 2015; 79: 180-185.

6. De CMP, Ferreira LM, Filho AN, Zafalon LF, Vicente HI, De SV. Comparison of methods for the detection of biofilm formation by Staphylococcus aureus isolated from bovine subclinical mastitis. Braz J Microbiol 2013; 44: 119-124. 
7. Guo JJ, Su MS, Wang LY, Ren JX, Yang M, Xie XM. Identification of dominant microbes from traditional Chinese medicine Pinelliae Rhizoma Fermentata. China J Chin Mater Med 2016; 41: 3027-3031.

8. Kim BY, Cho SJ, Kim HW, Kim SY, Lim SH, Kim KO, Choi CW, Lim CY, Byun BH, Cho Sl, et al. Genome wide expression analysis of the effect of Pinelliae Rhizoma extract on psychological stress. Phytother Res 2010; 24: 384-392.

9. Wu $X Y$, Zhao JL, Zhang $M$, Li F, Zhao $T$, Yang $L Q$. Sedative, hypnotic and anticonvulsant activities of the ethanol fraction from Rhizoma Pinelliae Praeparatum. J Ethnopharmacol 2011; 135: 325-329.

10. Ji $X$, Huang B, Wang G, Zhang C. The ethnobotanical, phytochemical and pharmacological profile of the genus Pinellia. Fitoterapia 2014; 93: 1-17.

11. Lee IA, Kim EJ, Kim DH. Inhibitory effect of betasitosterol on TNBS-induced colitis in mice. Planta Med 2012; 78: 896-898.

12. Chen J, Jiao R, Jiang Y, Bi Y, Chen ZY. Algal sterols are as effective as beta-sitosterol in reducing plasma cholesterol concentration. Journal of agricultural and food chemistry 2014; 62: 675-681.

13. Kim, KB, Kim MJ, Ahn DH. Lipase inhibitory activity of chlorophyll a, isofucosterol and saringosterol isolated from chloroform fraction of Sargassum thunbergii. Nat Prod Res 2014; 28: 1310-1312.

14. Wu Y, Lv S, Lian M, Wang C, Gao X, Meng Q. Study of characteristic aroma components of baked Wujiatai green tea by HS-SPME/GC-MS combined with principal component analysis. CyTA - Journal of Food 2016; 14: 1-10.

15. Martins SV, Madeira A, Lopes PA, Pires VM, Alfaia CM, Prates JA, Moura T, Soveral G. Adipocyte membrane glycerol permeability is involved in the anti-adipogenic effect of conjugated linoleic acid. Biochem Biophys Res Commun 2015; 458: 356-361.

16. Chaplin A, Parra P, Serra F, Palou A. Conjugated Linoleic Acid Supplementation under a High-Fat Diet Modulates Stomach Protein Expression and Intestinal Microbiota in Adult Mice. PLoS One 2015; 10: e0125091. 